\title{
Research on Wavelet-based Contourlet Transform Algorithm for Adaptive Optics Image Denoising
}

\author{
Li Dong-ming ${ }^{a, *}$, Zhang Li-juan ${ }^{\text {b,c }}$, Yang Jin-hua ${ }^{c}$, Su Weic \\ ${ }^{a}$ School of Information Technology, Jilin Agriculture University, Changchun, Jilin 130118, China \\ ${ }^{\mathrm{b}}$ College of Computer Science and Engineering, Changchun University of Technology, Changchun, Jilin \\ 130012, China \\ ${ }^{c}$ Changchun University of Science and Technology, Changchun, Jilin 130022, China \\ * Corresponding author. Tel: +86 13624499898 . \\ E-mail address: 1dm0214@163.com (D.-m. Li) .
}

\begin{abstract}
In this paper, we present a wavelet-based Contourlet transform (WBCT) method to adaptive optics (AO) image denoising. This method is implemented through combining with BayesShrink theory to estimate the threshold and then improving the adaptive method of selecting threshold, finally obtaining the optimal threshold. The WBCT transform coefficients of different decomposition scales and different direction to select the adaptive optimal threshold to achieve denoising. We evaluate our algorithm using the DWT-NABayesShrink algorithm, DTCWT-BayesShrink algorithm and CbATD algorithm as a benchmark. Using simulated and real observed AO images, we show that our approach with WBCT algorithm exhibits better performance both in peak signal-to-noise ratio (PSNR) and visual quality, which opens up many perspectives for AO image denoising in the astronautics field.
\end{abstract}

Keywords Image denoising; Contourlet transform (CT); Wavelet domain; BayesShrink; Adaptive Optics $(\mathrm{AO})$

\section{Introduction}

Because of the target background and the system's own factors, adaptive optics (AO) images contain a large amount of noise, which comes from the internal noise of sensor element, the quantization noise and transmission channel interference and so on. The noise will cause the target image is distorted seriously and impedes the target detection, tracking and positioning, so it is necessary to deal with the adaptive optics image denoising.

In recent years, many domestic and foreign scholars have proposed a number of denoising algorithms based on wavelet threshold. A universal threshold shrinkage method (VisuShrink) is proposed by Donoho and Johnstone [1], but with this algorithm the denoising image will appear ringing, too smooth, edge blur and the phenomena of lack of detail preservation. L.Kaur et al. [2] proposed a sub-band adaptive denoising method (NormalShrink). The method has the advantage of adaptive, according to different sub-bands of wavelet coefficients, it is possible to estimate the different threshold, however, the reservation of the details of the image still can not up to the requirements of denoising. Chang et al. [3] proposed adaptive wavelet threshold image denoising algorithm based on the principle of Bayesian (BayesShrink method). Contourlet transform proposed by Do and Vetterli [4] is a kind of multi-scale and multi-direction geometric transformation method, it inherits the multi-resolution of wavelet transform, and overcomes the problem of the nonlinear approximation of Ridge wave $k$ error decay order and the redundancy of the Curvelet transform. This paper combines with the BayesShrink theory, improves threshold adaptive 
selection method, uses the Wavelet-based Contourlet transform (WBCT), so an adaptive optical image denoising algorithm based on WBCT is proposed.

\section{Contourlet transform model based on wavelet domain}

Discrete Contourlet transform is composed of Laplacian Pyramid (LP) and Directional filter banks (DFB) to form a double layer filter bank structure, also known as the tower direction filter group [5]. The specific process of the transformation: first of all, image is multi-scale decomposed by LP transform, and the singular points are obtained. Then the singular points at the same direction are decomposed by the DFB to synthesize a coefficient. Finally, the final result is similar to the base structure of the contour segment to approximate the original image. The process is shown in Figure 1.

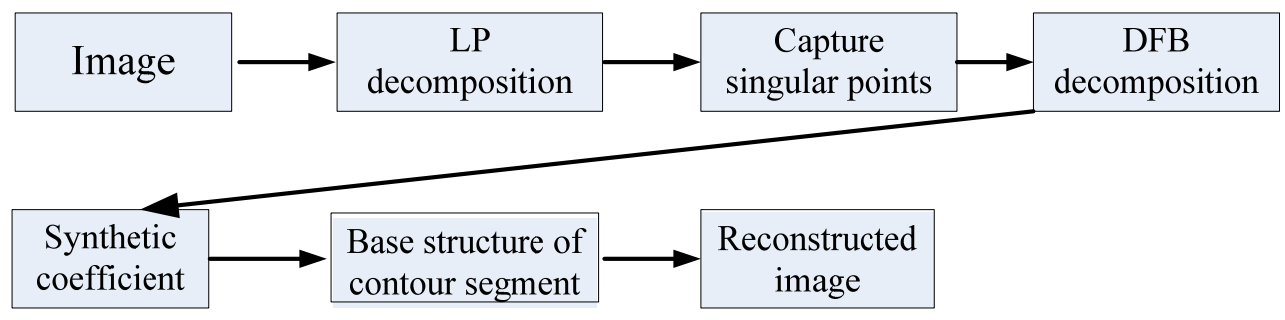

Fig.1 Contourlet transform process

In this paper, we propose a Contourlet transform based on wavelet technique. Specific process is to first generate four directional sub bands LL, HL, LH and HH by using two-dimensional discrete wavelet transform, and use the 12 directions by $1 \sim 12$ mark, WBCT has strict sampling data and contains the same size, which is in favor of image processing.

\subsection{The threshold calculation}

The additive noise model of AO image can be defined as follows:

$$
g(x, y)=f(x, y)+n(x, y)
$$

where $f(x, y)$ is the noise free signal, $g(x, y)$ is observation to the original signal, $n(x, y)$ is Gaussian white noise, whose mean is 0 and variance is $\sigma^{2}$. The aim of denoising is to restore the original image from the noisy image $g$ to maintain the image of $f$, and optimize the average variance.

In WBCT algorithm, select the threshold function and threshold are crucial. Threshold processing functions are commonly used in hard threshold and soft threshold function, and the soft threshold function is chosen in this paper. The soft threshold function is:

$$
\delta_{s}(x)=\left\{\begin{array}{c}
x-T, \quad x>T \\
x+T, \quad x<-T \\
0, \quad-T \leq x \leq T
\end{array}\right.
$$

In the formula, $T$ is the threshold. In this paper, we use the Bayesian estimation criterion of literature [4] to estimate the threshold, the expression is:

$$
T_{i, j}=\frac{\hat{\sigma}_{V}^{2}}{\hat{\sigma}_{x}}
$$

where $\hat{\sigma}_{V}^{2}$ is variance estimation of noise signal, $\hat{\sigma}_{x}$ is variance estimation of noise free signal. 
The WBCT transform to formula (1):

$$
C_{i, j}=X_{i, j}+V_{i, j}
$$

Where $C_{i, j}$ represent sub-band coefficients of the input image $\mathrm{g}(\mathrm{x}, \mathrm{y})$ of Contourlet transform decomposed. $X_{i, j}$ represents sub-band coefficients of the real image $\mathrm{f}(\mathrm{x}, \mathrm{y})$ of the sub-band coefficients after wavelet decomposition, $V_{i, j}$ represent sub-band coefficients of the noise $n(x, y)$ of Contourlet transform decomposed.

Because $f(x, y)$ and $n(x, y)$ are independent of each other, according to the formula (4):

$$
\sigma_{C}^{2}=\sigma_{X}^{2}+\sigma_{V}^{2}
$$

The noise variance $\sigma_{V}^{2}$ is estimated by the coefficient of the first sub-band HH1, the expression is:

$$
\hat{\sigma}_{V}^{2}=\frac{\operatorname{Median}\left(\left|C_{i, j}\right|\right)}{0.6745}, \quad C_{i, j} \in H H 1
$$

The experiment shows that the effect of BayesShrink denoising is improved, but assuming that the wavelet coefficients are independent, it has not considered the correlation between the coefficients. So we need to further improve this method.

\subsection{Adaptive threshold selection}

According to the central limit theorem, the coefficients $C_{i, j}$ of the sub bands in the Contourlet decomposition are subordinate to the generalized Gauss distribution, the estimated formula for the variance $\sigma_{C}^{2}$ of sub band noise image in BayesShrink denoising is:

$$
\hat{\sigma}_{C}^{2}=\frac{1}{m \times n} \sum_{k=1}^{m} \sum_{l=1}^{n}\left(C_{i, j}(k, l)\right)^{2}
$$

where $\sigma_{C}^{2}$ indicates that the variance of the sub-band $C_{i, j}, \hat{\sigma}_{C}^{2}$ is its estimation.

$\sigma_{C}^{2}$ depends on all the coefficients of the sub bands, in order to take into account the coefficients of locality and domain correlation, according to the characteristics of image to adaptive denoising, so we use WBCT to decompose the image, as far as possible to retain the image edge, texture and other details.

In this paper, the wavelet coefficients are processed by the neighborhood window, which is to estimate the noisy image variance of the current coefficients by calculating the variance of the noisy image within the window. Neighborhood window is the length $N$ of the square, the center is the current coefficient, per unit of $\mathrm{N}$ is an interval of adjacent wavelet coefficients on the vertical or horizontal direction, the value of $N$ can be $3 \times 3,5 \times 5,7 \times 7,9 \times 9$ etc $^{[6]}$. Assuming that in the size of the $m \times n$ sub band, the set of wavelet coefficients is $\left\{w_{p, q}\right\}$, the neighborhood window size is $N \times N$, the center position of the wavelet coefficient is $w_{p, q}$, the variance $\hat{\sigma}_{C, p, q}^{2}$ of the noisy image within the window is estimated to be:

$$
\hat{\sigma}_{C, p, q}^{2}=\frac{1}{N^{2}} \sum_{k, l=1}^{N}\left|C_{i, j}(k, l)\right|^{2}
$$


In the formula, $C_{i, j}(k, l)$ represents a collection of wavelet coefficients in the neighborhood window.

In the $m \times n$ sub bands, the neighborhood variance of noisy image estimation is defined as follows:

$$
\hat{\sigma}_{C}^{2}(L D)=\frac{1}{m \times n} \sum_{p=1}^{m} \sum_{q=1}^{n} \hat{\sigma}_{C, p, q}^{2}
$$

According to the formula (8) and (9), the neighborhood variance of the AO image is not dependent on all coefficients within the sub bands, but based on the neighborhood coefficients, which satisfies the local characteristics of the neighborhood. Therefore, in this method, the neighborhood variance $\hat{\sigma}_{C}^{2}(L D)$ of the free noisy image is calculated with the local correlation.

By formula (5), the neighborhood variance estimation $\hat{\sigma}_{X}^{2}(L D)$ of the noise free image can be obtained:

$$
\hat{\sigma}_{X}^{2}(L D)=\hat{\sigma}_{C}^{2}(L D)-\hat{\sigma}_{V}^{2}=\frac{1}{m \times n} \sum_{p=1}^{m} \sum_{q=1}^{n} \hat{\sigma}_{C, p, q}^{2}-\frac{\operatorname{Median}\left(\left|C_{i, j}\right|\right)}{0.6745}
$$

In order to prevent the occurrence of negative values of $\hat{\sigma}_{X}^{2}(L D)$, the formula (10) is further modified:

$$
\hat{\sigma}_{X}^{2}(L D)=\max \left(\left(\hat{\sigma}_{C}^{2}(L D)-\hat{\sigma}_{V}^{2}\right), 0\right)
$$

Modify the formula (3), the optimal threshold estimation for WBCT is obtained:

$$
T_{i, j}\left(\hat{\sigma}_{X}(L D)\right)=\frac{\hat{\sigma}_{V}^{2}}{\hat{\sigma}_{X}(L D)}=\frac{\hat{\sigma}_{V}^{2}}{\sqrt{\max \left(\left(\hat{\sigma}_{C}^{2}(L D)-\hat{\sigma}_{V}^{2}\right), 0\right)}}
$$

In this paper, the threshold of image denoising algorithm is based on the statistical analysis of the noisy image data, the WBCT transform coefficients of different decomposition scales $i$ and different direction $j$ to select the adaptive optimal threshold $T_{i, j}\left(\hat{\sigma}_{X}(L D)\right)$ to achieve denoising, the adaptive algorithm of WBCT transform is improved, which can make the observed AO image to get better denoising effect.

\section{AO image denoising algorithm based on WBCT}

The specific implementation steps of this algorithm are as follows:

Step 1: Multi-scale Contourlet transform based on wavelet domain for AO image, sub-band coefficients $C_{i, j}$ of the scale $i$ and direction $j$ are obtained;

Step 2: According to the formula (6) to estimate the noise variance $\hat{\sigma}_{V}^{2}$;

Step 3: $\quad$ The coefficients of $N$ sub bands are processed in the following(Except for low pass sub-band coefficients):

(1)For each of the WBCT coefficients, according to the formula (9) to calculate the variance of the corresponding neighborhood window containing image noise estimation $\hat{\sigma}_{C}^{2}(L D)$; 
(2) Calculate the average value of variance for the noise image with all coefficients, according to this value, the neighborhood variance of the Sub-band image with noise is estimated;

(3)According to the formula (12) to estimate the optimal threshold $T_{i, j}$ of sub-band coefficients $C_{i, j}$;

(4) The sub-band coefficients $C_{i, j}$ are applied to soft threshold function with formula (2), then the coefficients $\tilde{C}_{i, j}$ obtained after processing;

Step 4: WBCT inverse transform of the modified coefficient $\tilde{C}_{i, j}$ is used to obtain denoising AO image.

According to the formula (12), this algorithm considers the neighborhood local correlation in estimation of threshold, time complexity of the algorithm is $O(|g|)$, and the linear relationship between the observed noisy image $g$.

\section{Results and Analysis of AO image Denoising Experiments}

The objective evaluation criteria of the experimental results were measured by Peak Signal-to-noise ratio (PSNR) [7] and the calculation time, the PSNR unit is $\mathrm{dB}$, and the unit of calculation time is the second. Defined as

$$
P S N R=10 * \log _{10}\left[\frac{255^{2} \times M \times N}{\sum_{i=1}^{M} \sum_{j=1}^{N}[f(i, j)-\hat{f}(i, j)]^{2}}\right]
$$

In the formula, $M$ and $N$ represent the total number of pixels $x$-axis and $y$-axis on the image, respectively. $(i, j)$ represents a pixel point on image, $f(i, j)$ represents the gray value of the point $(i, j)$ on ideal image, and $\hat{f}(i, j)$ represents the gray value of the image being evaluated at point $(i, j)$.

\subsection{The Denoising Experiment on Simulate Images}

The test images are selected from the literature [8], and the three original images are selected, as shown in Fig.2. Fig.2 (a) is Fishing boats, and the image size is $512 \times 512$ pixels, 8 bits/pixel gray scale image. Fig.2 (b) is Lenna 256 level test pattern, and the image size is $512 \times 512$ pixels, 8 bits/pixel gray image. Fig. 2 (c) is Chemical plant, and the image size is $256 \times 256$ pixels, 8 bits/pixel gray image. The original images in Fig. 2 are added to the zero mean Gauss white noise, the noise standard deviation is 25, and the resulting noise images are shown in Fig.3. 


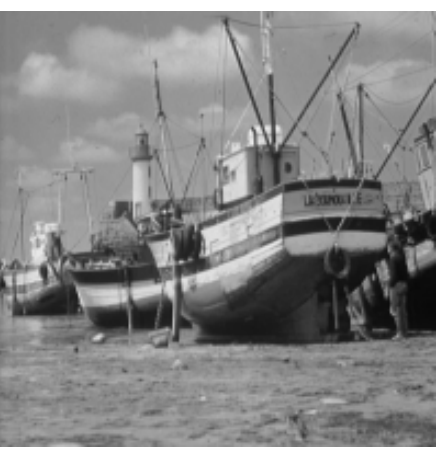

(a) Original image: Fishing Boat

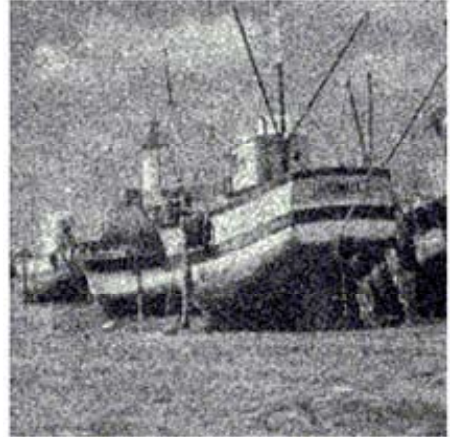

(a) Noise image: Fishing Boat, the noise standard deviation is 25

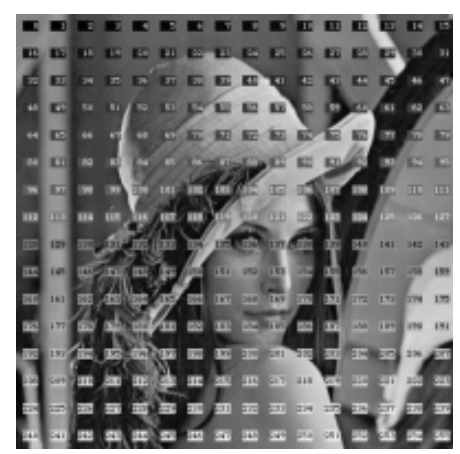

(b) Original image: 256 level test pattern

Fig.2 Three original images

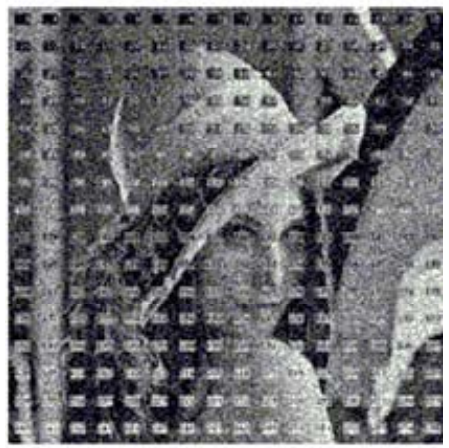

(b) Noise image: 256 level test pattern, the noise standard deviation is 25

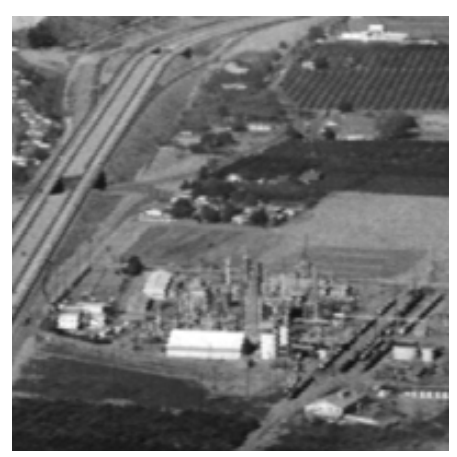

(c) Original image: Chemical plant

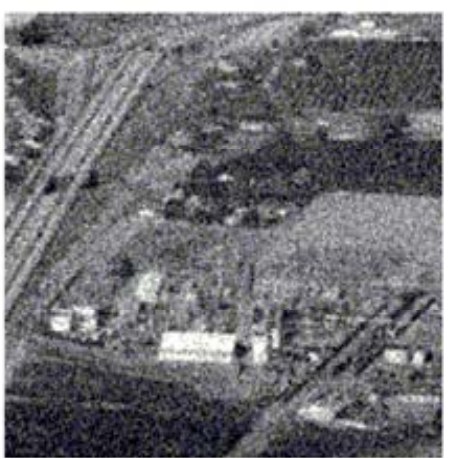

(c) Noise image: Chemical plant, the noise standard deviation is 25

Fig.3 Three noised images

In the experiment, we select four different sizes of the neighborhood windows, which are $3 \times 3,5 \times 5,7 \times 7$ and $9 \times 9$. The denoising experimental results based on the wavelet domain of the Bayesian Shrink for the PSNR value of denoising images are shown in Table 1. When the sizes of neighborhood windows are different, the PSNR values of the three denoising images are shown as Fig.4.

\begin{tabular}{|c|c|c|c|c|}
\hline $\begin{array}{c}\text { Noise standard } \\
\text { deviation }=25\end{array}$ & $\begin{array}{c}3 \times 3 \\
\text { PSNR(dB) }\end{array}$ & $\begin{array}{c}5 \times 5 \\
\text { PSNR(dB) }\end{array}$ & $\begin{array}{c}7 \times 7 \\
\text { PSNR(dB) }\end{array}$ & $\begin{array}{c}9 \times 9 \\
\text { PSNR(dB) }\end{array}$ \\
\hline Finshing Boat & 27.3508 & 28.3672 & 28.5817 & 28.7190 \\
\hline 256 level lenna & 26.7453 & 27.0365 & 27.1635 & 27.0875 \\
\hline Chemical plant & 28.0268 & 28.2772 & 28.6332 & 28.5211 \\
\hline
\end{tabular}

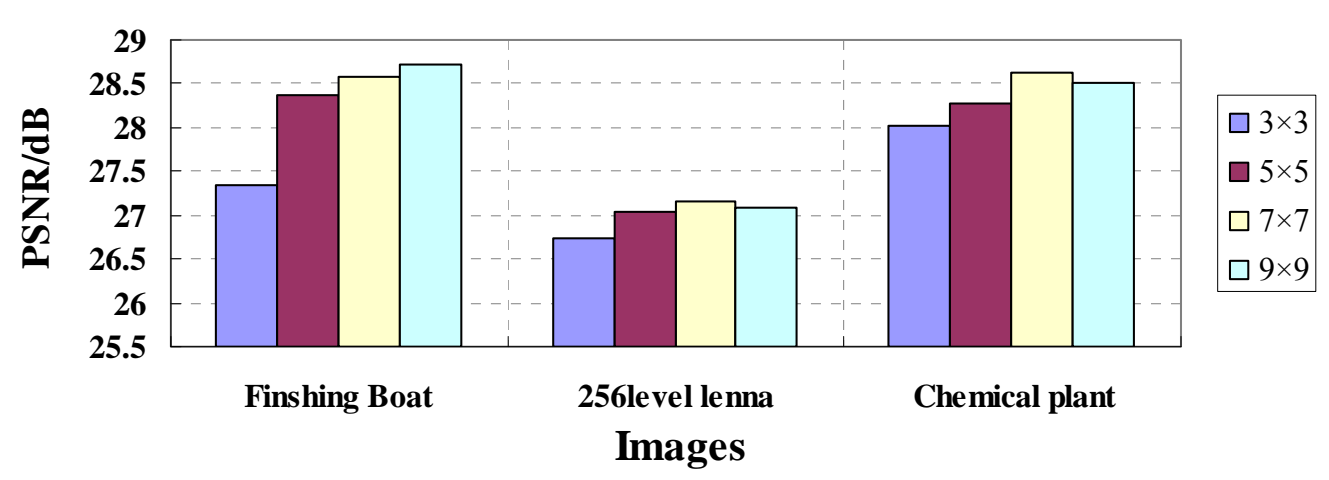

Fig.4 Comparison chart of the PSNR values for denoising images, when the different sizes of neighborhood windows 
From Table 1 and Fig.4, Finshing Boat image using local neighborhood window whose sizes are $7 \times 7$ and $9 \times 9$, denoising effect is best. 256 level lenna image and Chemical plant image use the local neighborhood window whose size is $7 \times 7$, the denoising effect is better than the other size of the neighborhood window of the denoising effect. Therefore, in this paper, we choose that the size of the local neighborhood window is $7 \times 7$.

In order to verify the validity of our algorithm, we compare the three algorithms of DWT-NABayesShrink ${ }^{[9]}$, CbATD ${ }^{[10]}$ and DTCWT-BayesShrink ${ }^{[11]}$, which is shown in Table 2. It is shown from Table 2 that our algorithm can obtain a high PSNR, so our proposed algorithm can be effectively exploited to the noise removal in images. But from the calculation time of the experimental results, the computation load of our algorithm is slightly larger than the other three kinds of denoising algorithms, so our future work will further improve the performance of the algorithm.

Table 2 PSNR (dB) data and calculation time comparison of different denoising algorithms as $25 \mathrm{~dB}$ noise level

\begin{tabular}{|c|c|c|c|c|c|c|c|c|}
\hline \multirow{2}{*}{$\begin{array}{l}\text { Image } \\
\text { name }\end{array}$} & \multicolumn{2}{|c|}{ DWT-NABayesShrink } & \multicolumn{2}{|c|}{ CbATD } & \multicolumn{2}{|c|}{ DTCWT-BayesShr ink } & \multicolumn{2}{|c|}{ Our algorithm } \\
\hline & PSNR (dB) & $\begin{array}{l}\text { Calculatio } \\
\mathrm{n} \text { time (s) }\end{array}$ & $\begin{array}{l}\text { PSNR (d } \\
\text { B) }\end{array}$ & $\begin{array}{c}\text { Calculation } \\
\text { time (s) }\end{array}$ & PSNR (dB) & $\begin{array}{c}\text { Calculation } \\
\text { time (s) }\end{array}$ & $\begin{array}{l}\text { PSNR (dB } \\
\text { ) }\end{array}$ & $\begin{array}{l}\text { Calculatio } \\
\text { n time (s) }\end{array}$ \\
\hline $\begin{array}{c}\text { Finshing } \\
\text { Boat }\end{array}$ & 30.0985 & 2.6 & 31.5998 & 3.1 & 31.7856 & 3.4 & 32.01452 & 3.9 \\
\hline $\begin{array}{c}256 \text { level } \\
\text { lenna }\end{array}$ & 33.9765 & 3.5 & 34.2506 & 3.7 & 34.2162 & 3.6 & 34.8725 & 3.8 \\
\hline $\begin{array}{c}\text { Chemical } \\
\text { plant }\end{array}$ & 32.8603 & 2.9 & 33.6633 & 3.4 & 33.4983 & 3.7 & 34.1092 & 3.6 \\
\hline
\end{tabular}

Fig.5 is PSNR experimental results to measure the parameters of contrast histogram, and the denoising algorithms including DWT-NABayesShrink, CbATD, DTCWT-BayesShrink and our algorithm. From Fig. 5 can be known, our algorithm proposed in this paper has a high PSNR, which is applied to the three images. This shows that the proposed denoising algorithm can effectively remove the noise.

PSNR values comparison of four denoising algorithms

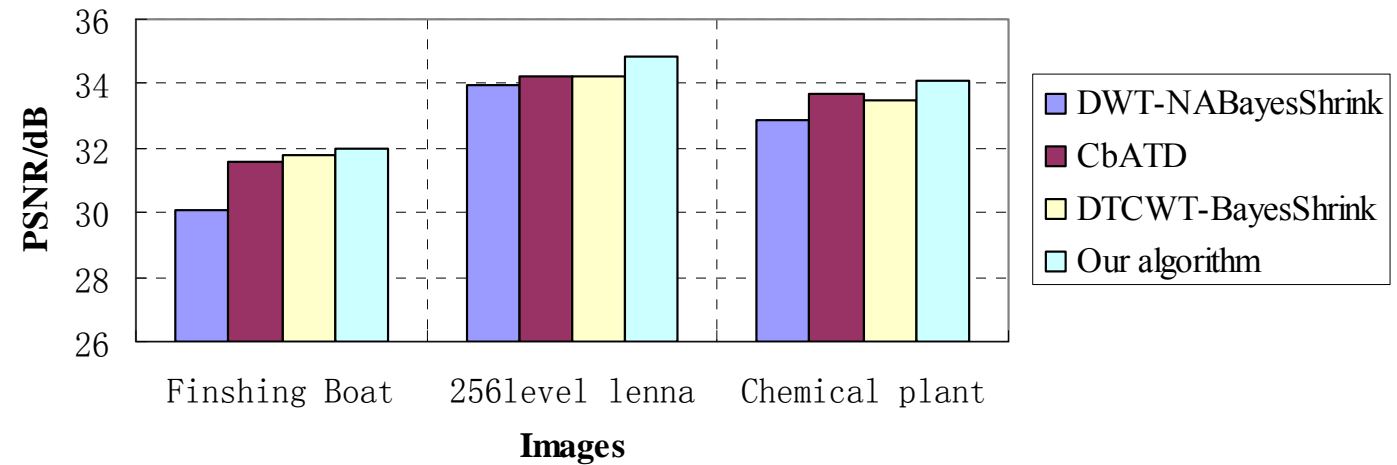

Fig.5 Comparison of PSNR values for three different noise images by four denoising algorithms 
Fig.6 is Calculation time of experimental results to measure the parameters of contrast histogram, for three different noise images by four denoising algorithms, which are DWT-NABayesShrink, CbATD, DTCWT-BayesShrink and our algorithm. It can be seen from Fig.6 that our proposed algorithm for the three images denoising, whose computation is slightly larger than the other three denoising algorithms. But our denoising images with higher PSNR than the other three kinds of algorithms for image denoising.

\section{Calculation time comparison of four denoising algorithms}

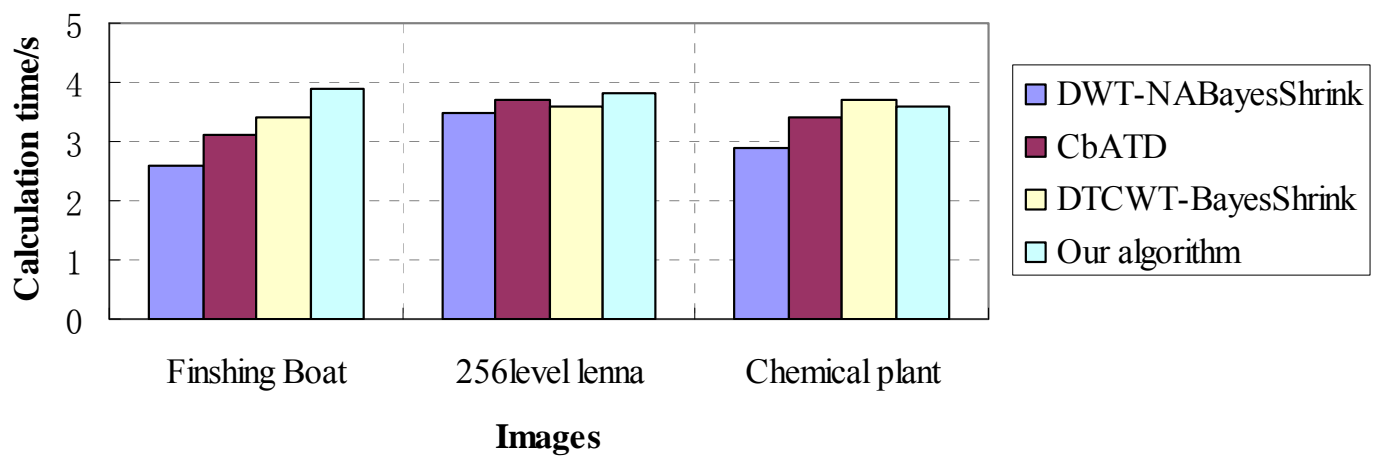

Fig.6 Comparison of calculation time for three different noise images by four denoising algorithms

In order to verify the effectiveness of our algorithm for denoising, the noise level is Chemical plant $25 \mathrm{~dB}$ image as an example, and the comparison of the four algorithms for image denoising visual effects, which is shown in Fig.7.

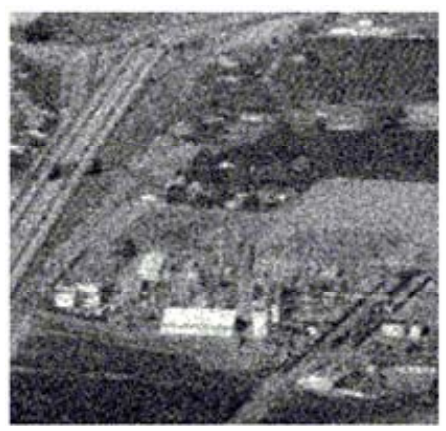

(a) Noise image of Chemical Plant, standard deviation is 25

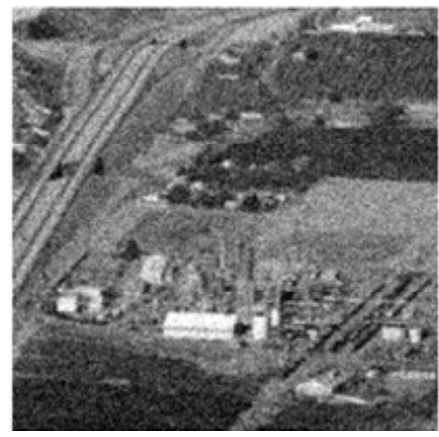

(b) DWT-NABayesShrink

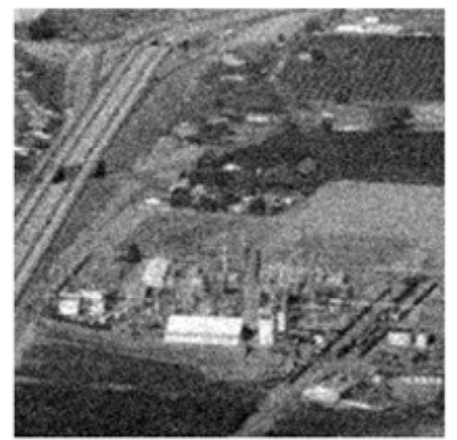

(c) CbATD

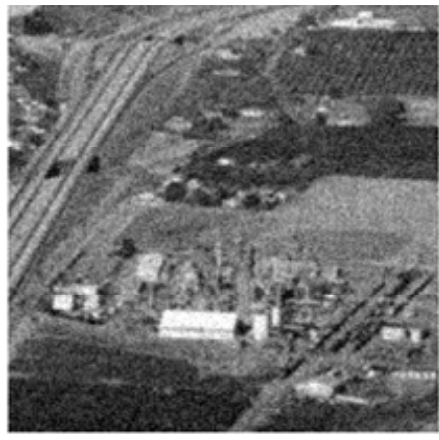

(d) DTCWT-BayesShrink

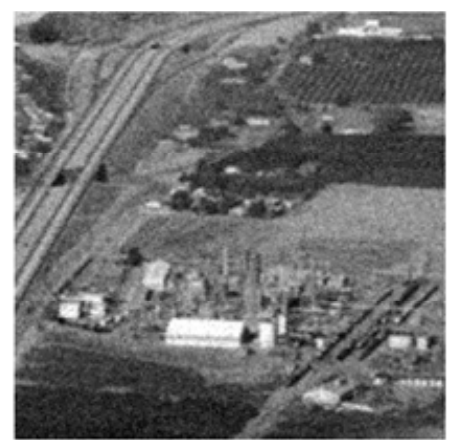

(e) Image denoised by our algorithm

Fig.7 Denoising contrast effect of different denoising algorithms on Chemical plant image with noise level of $25 \mathrm{~dB}$ 


\subsection{Denoising Experiments about AO Image}

The experiment for $\mathrm{AO}$ image denoising without reference images is carried out by the algorithm proposed in this paper. The observed AO images for experiments were taken by the $1.2 \mathrm{~m}$ AO telescope from Chinese Academy of Sciences in Yunnan observatory on December 3, 2006. The size for imaging CCD is $320 \times 240$ pixel array, the atmospheric coherence length $\mathrm{r}_{0}=13 \mathrm{~cm}$, optical imaging focal length $l=20 \mathrm{~m}$. AO images' denoising experiments based on DWT-NABayesShrink algorithm, CbATD algorithm, DTCWT-BayesShrink algorithm and the proposed algorithm proposed in this paper are compared.

Fig. 8 is the observed multi-frame degraded AO images, where Fig.8 (a), 8(b), 8 (c) and 8(d) are for the observation of the AO image (in order to save space, we show only four frames, and the rest is omitted). Fig.9 is the comparison results of denoising images based on four kinds of algorithms. Fig. 9(a) is the restored image based on DWT-NABayesShrink algorithm, PSNR $=24.12313 \mathrm{~dB}$; Fig. 9(b) is the CbATD algorithm restored image, PSNR= $23.5691 \mathrm{~dB}$; Fig. 9(c) is DTCWT-BayesShrink algorithm restored image, PSNR $=23.5890 \mathrm{~dB}$; Fig. 9 (d) is the restored image based on the method proposed in this paper, $\mathrm{PSNR}=26.2072 \mathrm{~dB}$, the size of the local neighborhood window is $7 \times 7$. Table 3 is the PSNR data and Calculation time data of comparison results for four denoising algorithms about the AO images denoising.

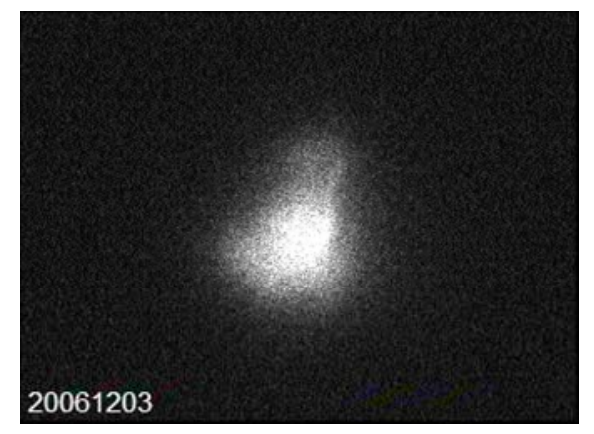

(a) The degraded AO image frame 1

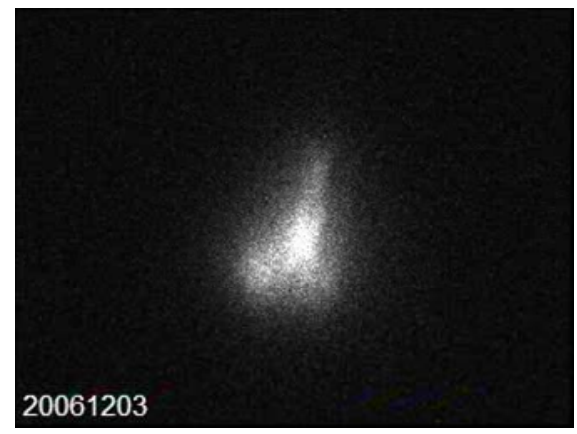

(c) The degraded AO image frame 3

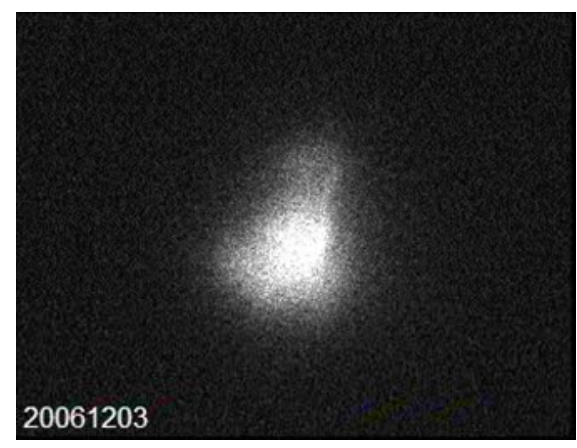

(b) The degraded AO image frame 2

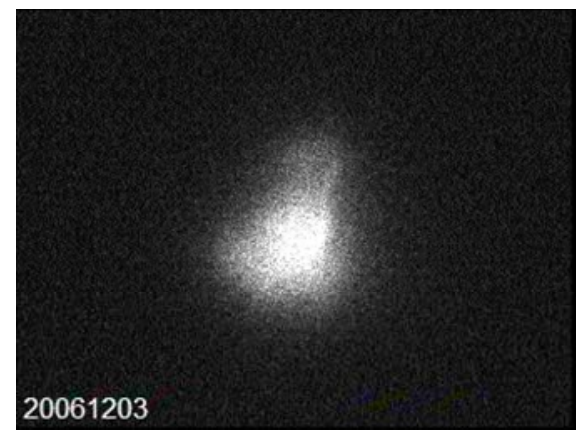

(d) The degraded AO image frame 4

Fig. 8 The multi-frame observed AO images 


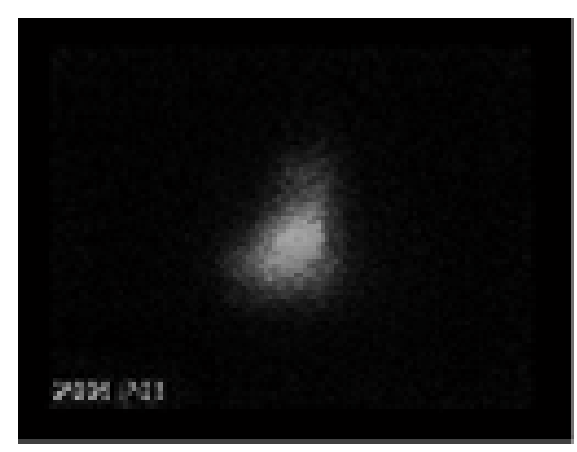

(a) DWT-NABayesShrink algorithm

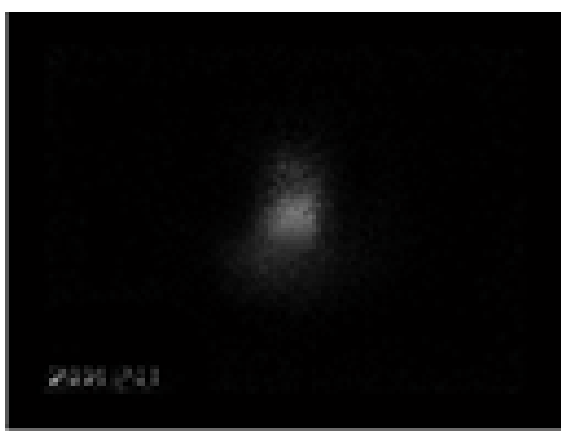

(c) DTCWT-BayesShrink algorithm

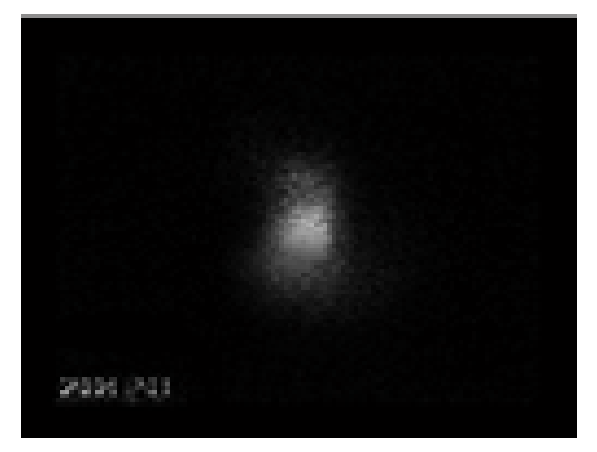

(b) CbATD algorithm

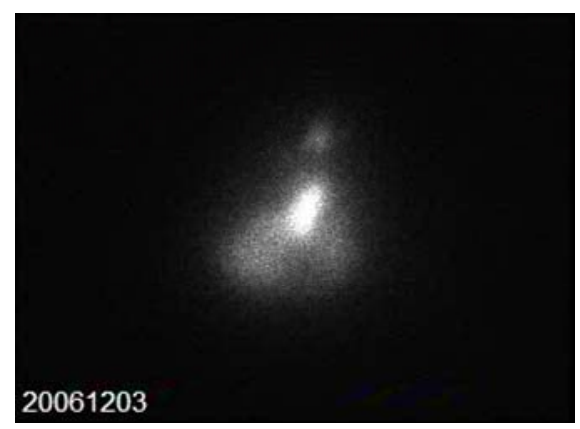

(d) Our algorithm

Fig.9 Comparison results of denoising images based on four algorithms

From the above experimental results, it shows that the proposed algorithm in this paper can reduce the fuzzy degree of AO image. The restoration effect is improved obviously, and the false contour is obviously reduced. But its disadvantage is time consumption, it needs many times of iteration to get a better restoration result.

Table 3 The PSNR data and calculation time data of comparison results for four denoising algorithms about the AO images denoising

\begin{tabular}{cllccccccc}
\hline & \multicolumn{2}{c}{ DWT-NABayesShrink } & \multicolumn{2}{c}{ CbATD } & \multicolumn{2}{c}{ DTCWT-BayesShrink } & \multicolumn{2}{c}{ Our algorithm } \\
\cline { 2 - 10 } Image & PSNR & Calculatio & PSNR & Calculat & PSNR & Calculation time $(\mathrm{s})$ & PSNR & Calculatio \\
& $(\mathrm{dB})$ & $\mathrm{n}$ time $(\mathrm{s})$ & $(\mathrm{dB})$ & $\begin{array}{c}\text { ion } \\
\text { time }(\mathrm{s})\end{array}$ & $(\mathrm{dB})$ & & & $(\mathrm{dB})$ & $\mathrm{n}$ time(s) \\
AO Image & 24.12313 & 4.0 & 23.5691 & 3.9 & 23.5890 & 4.3 & 26.2072 & 4.2 \\
\hline
\end{tabular}

Therefore, the biggest advantage of Contourlet transform and wavelet transform combined is highly anisotropic, can better express the edge information of images. From the experimental results, the proposed denoising algorithm in this paper that is wavelet-based Contourlet transform algorithm has strong adaptability, and the calculation time is slightly larger than the other denoising algorithms. The advantage of our algorithm for image denoising not only has a higher peak signal to noise ratio, but also has a better visual effect, especially for the edge restoration and linear structure weak situation is particularly effective.

\section{Conclusions}

In this paper, according to the characteristic of adaptive optics image, we introduce the idea of multi-scale and multi-direction Contourlet transform into the adaptive optics image denoising process. Firstly, we establish a Contourlet transform model based on wavelet domain, and on this basis, combine 
with the BayesShrink theory, image adaptive threshold denoising algorithm based on Contourlet transform in wavelet domain is designed. Finally, the adaptive algorithm of WBCT transform is improved, and we give the specific implementation steps of this algorithm. Experimental results of the objective evaluation criteria using the peak signal to noise ratio and the calculation time to measure. During the experiment, we choose the size of local neighborhood window as $7 \times 7$. We compare our algorithm with the DWT-NABayesShrink algorithm, CbATD algorithm and DTCWT-BayesShrink algorithm and show that our approach outperforms the others. The experimental results presented show that our algorithm based on Contourlet transform in wavelet domain has strong adaptability. The image process of AO observed images show that the restoration quality is better than the other algorithms. However, the calculation time is slightly larger than the other denoising algorithms. The next work is to introduce the observation equipment information of the $\mathrm{AO}$ image and other prior knowledge into the calculation of the solution, in order to improve computing power. The results of actual $\mathrm{AO}$ image restoration have important application value.

\section{References}

[1] D. Donoho, I. M. Johnstone. Ideal Spatial Adaptation by Wavelet Shrinkage [J]. Biometrika, 1994, 36(8):425-450

[2] Kaur L, Gupta S, Chauhan R.C. Image Denoising Using Wavelet Thresholding [J]. Computer Vision, Graphics and Image Processing, 2002 http://www.ee.iitb.ac.in/-icvgip/PAPERS/202.pdf

[3] S. G. Chang, B.Yu, Martin Vetterli. Adaptive Wavelet Thresholding for Image Denoising and Compression[J]. IEEE Trans Image Processing, 2000, 9(9):1535-1545

[4] M.N.Do, M.Vetterli. The Contourlet Transform: an Efficient Directional Multi-resolution Image Representation [J]. IEEE Transactions on Image Processing, 2005,14(12):2091-2106

[5] Zhang Jingjing, Fang Yonghua. Novel Denoising Method for Remote Sensing Image Based on Contourlet Transform [J]. Acta Optica Sinica, 2008, 28(3): 462-466

[6] Zhang Wenwen. Research on Image Denoising Algorithm Based on Transform Domain [D]. Hangzhou: Zhejiang University, 2013: 15-25.

[7] M.R. Banham, A. K. Katsagellos. Digital Image Restoration[J]. IEEE Signal Processing Mag., 1997, 14: $24-41$

[8] USC-SIPI Image Database [DB/OL].[2016-1-12] http://sipi.usc.edu/database

[9] Gong Xiaolin, Mao Ruiquan, Liu Kaihua. Threshold Denoising Method for Wavelet Image Based on Adaptive Neighborhood Coefficient[J]. Computer Engineering, 2010, 36(11): 206-208

[10] Chen Bo.The Theory and Algorithms of Adaptive Optics Image Restoration [D]. Zhengzhou Information Engineering University, 2008.

[11] Yang Guodong, Yan Qianshi. Denoising Technology for the Dual-tree Complex Wavelet Image Based on Bayesian Estimation[J]. Journal of Xi'an Polytechnic University, 2009, 23(3): 75-98.

\section{Acknowledgements}

This research is supported by the National Science Foundation of China (Grant No.61402193), the Scientific and Technological Research Project of Jilin Province Department of Education (Grant No. 2013145, No. 201363), Department of Education of Jilin Province "Twelfth Five Year" Scientific and Technological (Grant No.2015410). 